\title{
Abnormally Large Baseline P300 Amplitude Is Associated With Conversion to Psychosis in Clinical High Risk Individuals With a History of Autism: A Pilot Study
}

OPEN ACCESS

Edited by:

Noah James Sasson,

The University of Texas at Dallas,

United States

Reviewed by:

Wei Yanyan,

Shanghai Jiao Tong University, China

Clément Dondé,

Université Grenoble Alpes, France

Tineke Grent-'T-Jong,

University of Glasgow,

United Kingdom

*Correspondence:

Jennifer H. Foss-Feig

jennifer.foss-feig@mssm.edu

${ }^{\dagger}$ Deceased

Specialty section:

This article was submitted to

Schizophrenia,

a section of the journal

Frontiers in Psychiatry

Received: 03 August 2020 Accepted: 05 January 2021

Published: 09 February 2021

Citation:

Foss-Feig JH, Guillory SB, Roach BJ, Velthorst $E$, Hamilton $H$, Bachman $P$,

Belger A, Carrion R, Duncan E,

Johannesen J, Light GA,

Niznikiewicz M, Addington JM,

Cadenhead KS, Cannon TD,

Cornblatt B, McGlashan T, Perkins D,

Seidman LJ, Stone WS, Tsuang M,

Walker EF, Woods S, Bearden CE and Mathalon DH (2021) Abnormally Large

Baseline P300 Amplitude Is

Associated With Conversion to

Psychosis in Clinical High Risk Individuals With a History of Autism: A

Pilot Study.

Front. Psychiatry 12:591127.

doi: 10.3389/fpsyt.2021.591127
Jennifer H. Foss-Feig ${ }^{1 *}$, Sylvia B. Guillory ${ }^{1}$, Brian J. Roach ${ }^{2}$, Eva Velthorst ${ }^{1}$, Holly Hamilton ${ }^{2,3}$, Peter Bachman ${ }^{4}$, Aysenil Belger ${ }^{5}$, Ricardo Carrion ${ }^{6}$, Erica Duncan ${ }^{7}$, Jason Johannesen ${ }^{8}$, Gregory A. Light ${ }^{9}$, Margaret Niznikiewicz ${ }^{10}$, Jean M. Addington ${ }^{11}$, Kristin S. Cadenhead ${ }^{9}$, Tyrone D. Cannon ${ }^{8}$, Barbara Cornblatt ${ }^{6}$, Thomas McGlashan ${ }^{8}$, Diana Perkins ${ }^{5}$, Larry J. Seidman ${ }^{10 t}$, William S. Stone ${ }^{10}$, Ming Tsuang ${ }^{9}$, Elaine F. Walker ${ }^{7}$, Scott Woods ${ }^{8}$, Carrie E. Bearden ${ }^{12}$ and Daniel H. Mathalon ${ }^{2,3}$

${ }^{1}$ Department of Psychiatry and Seaver Autism Center for Research and Treatment, Icahn School of Medicine at Mount Sinai, New York, NY, United States, ${ }^{2}$ San Francisco VA Health Care System, San Francisco, CA, United States, ${ }^{3}$ Department of Psychiatry, University of California, San Francisco, San Francisco, CA, United States, ${ }^{4}$ Department of Psychiatry, University of Pittsburgh, Pittsburgh, PA, United States, ${ }^{5}$ Department of Psychiatry, University of North Carolina, Chapel Hill, NC, United States, ${ }^{6}$ Department of Psychiatry, Zucker Hillside Hospital, New York, NY, United States, ${ }^{7}$ Departments of Psychology and Psychiatry, Atlanta VA Health Care System and Emory University, Decatur, GA, United States, ${ }^{8}$ Departments of Psychology and Psychiatry, Yale University, New Haven, CT, United States, ${ }^{9}$ Department of Psychiatry, University of California, San Diego, San Diego, CA, United States, ${ }^{10}$ Department of Psychiatry, Harvard University, Cambridge, MA, United States, ${ }^{11}$ Department of Psychiatry, University of Calgary, Calgary, AB, Canada, ${ }^{12}$ Departments of Psychiatry and Biobehavioral Sciences and Psychology, University of California, Los Angeles, Los Angeles, CA, United States

Psychosis rates in autism spectrum disorder (ASD) are 5-35\% higher than in the general population. The overlap in sensory and attentional processing abnormalities highlights the possibility of related neurobiological substrates. Previous research has shown that several electroencephalography (EEG)-derived event-related potential (ERP) components that are abnormal in schizophrenia, including P300, are also abnormal in individuals at Clinical High Risk (CHR) for psychosis and predict conversion to psychosis. Yet, it is unclear whether P300 is similarly sensitive to psychosis risk in help-seeking CHR individuals with ASD history. In this exploratory study, we leveraged data from the North American Prodrome Longitudinal Study (NAPLS2) to probe for the first time EEG markers of longitudinal psychosis profiles in ASD. Specifically, we investigated the P300 ERP component and its sensitivity to psychosis conversion across CHR groups with (ASD+) and without (ASD-) comorbid ASD. Baseline EEG data were analyzed from $304 \mathrm{CHR}$ patients (14 ASD+; 290 ASD-) from the NAPLS2 cohort who were followed longitudinally over two years. We examined P300 amplitude to infrequent Target (10\%; P3b) and Novel distractor (10\%; P3a) stimuli from visual and auditory oddball tasks. Whereas P300 amplitude attenuation is typically characteristic of $\mathrm{CHR}$ and predictive of conversion to psychosis in non-ASD sample, in our sample, history of ASD moderated this relationship such that, in CHR/ASD+ individuals, enhanced - rather than attenuated - visual P300 (regardless of stimulus type) was associated with psychosis conversion. This pattern was also seen for auditory P3b amplitude to Target stimuli. Though drawn from a small sample of $\mathrm{CHR}$ individuals with $\mathrm{ASD}$, these preliminary results point to a paradoxical 
effect, wherein those with both CHR and ASD history who go on to develop psychosis have a unique pattern of enhanced neural response during attention orienting to both visual and target stimuli. Such a pattern stands out from the usual finding of P300 amplitude reductions predicting psychosis in non-ASD CHR populations and warrants follow up in larger scale, targeted, longitudinal studies of those with ASD at clinical high risk for psychosis.

Keywords: autism spectrum disorder, psychosis, P300, EEG, conversion, prodrome

\section{INTRODUCTION}

While autism spectrum disorder (ASD) and the schizophrenia spectrum disorders (SCZ) are considered diagnostically distinct, they share phenotypic features, genetic overlap, and a common historical background $(1,2)$ that highlight the possibility of related neurobiological substrates. As a neurodevelopmental disorder, ASD diagnosis -characterized by impaired social interaction and communication, alongside repetitive and restricted behaviors and interests (3) occurs in early childhood. SCZ is also characterized by impairments in social interactions, but hallmark symptoms of delusions, hallucinations, disorganized thought and behavior, and a constellation of negative symptoms, typically emerge in late adolescence and early adulthood (4). Yet, epidemiological studies also point to considerable overlap between the two disorders. Estimates of SCZ rates in ASD, for example, are 5-35\% higher than in the general population (5-7), while rates of ASD diagnoses in SCZ patients range from $<1-52 \%$ (8). Importantly, prodromal symptoms of SCZ that precede full-blown illness also include social deficits (9) that share some overlap with core features of ASD. In addition, cognitive deficits are pervasive in both ASD and SCZ, as well as in prodromal SCZ. Indeed, tasks that probe attention, memory, and executive functioning find differences in processing speed, accuracy, and perceptual discrimination/detection thresholds compared to typically developing (TD) control cohorts across disorders (10-12).

Recent trends in the schizophrenia field have focused on examining clinical and neurobiological characteristics in individuals at clinical high risk for psychosis (CHR) in order to identify which features are most predictive of transition to full-blown psychosis. Clinically, some of the best predictors of conversion to psychosis include genetic risk, history of substance abuse, and severity of social impairment (13). Neurologically and neuropsychologically, brain volume abnormalities $(14,15)$, reduced processing speeds and worse verbal memory (16) are associated with increased risk and an earlier psychosis conversion in CHR individuals. Until recently, it was unknown whether individuals with ASD who presented at CHR services showed similar prodromal features and conversion rates to those seen in the broader CHR general population. However, a recent study from the second wave of the North American Prodrome Longitudinal Study (NAPLS2) revealed that CHR individuals with prior ASD diagnoses had more social impairment than other CHR individuals, but similar positive symptoms of psychosis and similar rates of converting to co-morbid psychotic illness (17).
However, it is not yet known whether neurological profiles and predictors of conversion to psychosis are similar between CHR individuals with and without ASD.

Event-related potentials (ERPs) have been widely used in understanding altered information processing in clinical vs. nonclinical populations. In SCZ, reduced P300 amplitude during detection of an infrequent target stimulus is among the most reliable and replicable findings (18-20). P300 is a positive-going ERP associated with shifting and allocation of attention, as well as stimulus salience (21-27), where larger amplitudes reflect larger resource allocation toward these processes. The robust amplitude reduction in SCZ suggest that the P300 might be a possible biomarker for the illness (28). Moreover, attenuated P300 is also seen in CHR individuals $(29,30)$ and may be useful as a predictive tool when identifying individuals at risk for psychosis conversion (31-33) and considering preventative interventions.

P300 can be divided into two subcomponents: P3a and P3b. P3a is maximal over frontocentral scalp and reflects attention orienting toward novel stimuli that are not behaviorally-relevant, in other words, distractors requiring no response (34-38). P3b, on the other hand, is maximal over central-parietal scalp and reflects allocation of attention toward infrequent stimuli that require behavioral response. In schizophrenia, P3b amplitude deficits are well-replicated, particularly in the auditory modality $(18,19,39-43)$. Auditory P3a amplitude deficits have also been detected $(19,29,42,44-50)$, though they may be less robust $(44,51,52)$. In CHR, both P3a and P3b amplitude reductions have been identified $(29,46,50,53-60)$, with emerging evidence that auditory $\mathrm{P} 3 \mathrm{~b}$ amplitude may be predictive of conversion to psychosis $(33,60)$.

The P300 literature in ASD is less clear than in SCZ and CHR. A recent meta-analysis of the $\mathrm{P} 3 \mathrm{a}$ and $\mathrm{P} 3 \mathrm{~b}$ found only reduction in P3b amplitude to be a reliable alteration, whereas P3b latency and both P3a amplitude and latencies were generally similar to controls (61). Clear differences in P300 response to auditory vs. visual stimuli have not been reported, though in general there are more systematic findings of impaired auditory processing and enhanced visual perceptual functioning in the ASD literature broadly. Whether there is a particular pattern of P300 alterations that characterizes individuals with ASD and CHR or predicts who in this population will develop full-blown psychotic illness has not been examined.

The present study leveraged a large, longitudinal study of CHR individuals to examine whether the neural profile and predictors of conversion to psychosis are comparable between individuals with and without co-morbid ASD. In particular, we 
focused on early attention-modulated indices in response to both attended (P3b) and task-irrelevant (P3a) sensory input. By testing both auditory and visual sensory modalities, we further examined whether, as in CHR more generally, sensory domain affects the predictive utility of brain-based measures dependent on ASD status. We hypothesized that whereas consistent P300 amplitude attenuations are predictive of conversion to psychosis in general CHR populations, P3b deficits may be more specific in those with ASD history and visual P300 deficits may be lacking regardless of conversion. Because there have been no longitudinal studies of neural markers of psychosis risk and development in ASD, this study capitalized on a large-scale study in order to identify a rare subset of individuals with both ASD and CHR. Though our sample size is small and our findings preliminary, this exploratory work offers the first window into brain-based predictors of psychosis conversion in individuals with ASD and a launching point for future, larger studies.

\section{METHOD}

\section{Participants}

EEG data were available from the baseline visits of 304 patients who participated in the North American Prodrome Longitudinal Study (NAPLS2) (62), a consortium of eight research centers studying CHR between 2009 and 2013, comprising help-seeking individuals ages $12-35$ years, observed for up to $2 \frac{1}{2}$ years. These patients represent a subset of the full NAPLS2 cohort who completed both the auditory and visual oddball tasks (see below) at baseline and either converted to psychosis anytime within the 24-mo follow-up period or were followed through to the 24-month visit without converting. All CHR individuals met one or more of the three Criteria of Prodromal Syndromes (COPS): attenuated positive symptom syndrome (APSS), genetic risk and deterioration (GRD), and/or brief intermittent psychotic syndrome (BIPS). APSS requires at least one attenuated positive psychotic symptom, begun or worsened in the past year, and of insufficient severity to meet diagnostic criteria for a psychotic disorder. GRD is defined in NAPLS2 as a combination of functional decline (30\% or greater drop in Global Assessment of Function score over the month preceding the baseline visit, as compared to 12 months prior) and genetic risk, defined as either schizotypal personality disorder or a first-degree relative with a schizophrenia spectrum disorder. BIPS reflects the presence of a one or more positive psychotic symptom meeting severity threshold but too brief to meet diagnostic criteria for psychosis (63). There was no formal testing or screening for peripheral sensory deficits as part of study procedures or exclusion criteria.

For this study, CHR participants were grouped based whether or not they had a comorbid ASD diagnosis noted at baseline (ASD+: comorbid ASD; ASD-: no ASD) to predict whether they converted to psychosis (Conv+: converter; Conv-: non-converter) within the 2 years following their baseline visit. All patients in the ASD+ group met DSM-IV criteria for Autistic Disorder, Asperger's Disorder, or Pervasive Developmental Disorder-Not Otherwise Specified (PDD-NOS) using a combination of DSM-IV checklist during baseline clinical interview, medical records, and caregiver report of historical diagnosis. All patients designated as Conv+ experienced conversion from CHR state to psychosis, determined by meeting the Structured Interview for Psychosis-Risk Syndromes (SIPS) $(64,65)$, Presence of Psychotic Symptoms criteria (13). Conversion decisions were discussed and approved on a weekly consensus call. In total, of the 304 participants with included data, 290 did not have ASD (ASD-) and 14 had previous ASD history (ASD+). Within the ASD- group, 71 converted to psychosis (Conv+); conversion to psychosis occurred in four participants within in the ASD+ group. Table 1 summarizes demographic information and assessment scores by group. The sample yielded closely age-matched groups (Main Effect Conversion: $\mathrm{F}_{1,300}$ $=0.61, p=0.44$; Main Effect ASD: $\mathrm{F}_{1,300}=3.69, p=$ 0.056; Conversion $\times$ ASD Interaction: $\mathrm{F}_{1,300}=0.042, p=0.84$ ). Illness level also did not differ among groups at baseline. In particular, across SIPS positive, negative, disorganization, and general subscales, there were no main effects of conversion status (Positive: $\mathrm{F}_{1,298}=0.60, p=0.44$; Negative: $\mathrm{F}_{1,300}=0.24, p=$ 0.68; Disorganization: $\mathrm{F}_{1,299}=0.037, p=0.85$; General: $\mathrm{F}_{1,298}$ $=0.24, p=0.88$ ) or ASD diagnosis (Positive: $\mathrm{F}_{1,300}=0.14$, $p=0.71$; Negative: $\mathrm{F}_{1,298}=0.017, p=0.90$; Disorganization: $\mathrm{F}_{1,299}=0.45, p=0.50$; General: $\mathrm{F}_{1,298}=0.48, p=0.49$ ), and no significant interaction effects between conversion status and ASD (Positive: $F_{1,300}=1.96, p=0.16$; Negative: $F_{1,298}=0.97, p$ $=0.22$; Disorganization: $\mathrm{F}_{1,299}=1.48, p=0.23$; General: $\mathrm{F}_{1,298}$ $=0.072, p=0.79$ ) (see Table 1 ).

The Institutional Review Boards of the eight participating sites approved all study protocols. All adult subjects gave informed consent. Minor subjects provided verbal assent while their parents/guardians provided written informed consent.

\section{Oddball Paradigm}

The experiment consisted of two (visual, auditory) threestimulus oddball paradigms, where in addition to the frequent, standard stimulus and the rare, target stimulus, there were also rare novel, task-irrelevant stimuli (37). Each oddball task (i.e., visual and auditory) comprised three blocks of 150 trials, of which $80 \%$ of trials were standards (visual: small blue circle presented at the vertical and horizontal meridian; auditory: $500 \mathrm{~Hz}, 50 \mathrm{~ms}$ tone with a $5 \mathrm{~ms}$ rise/fall time at $62 \mathrm{~dB}$ ). An additional $10 \%$ of trials were target stimuli (visual: large blue circle presented at the vertical and horizontal meridian; auditory: $1,000 \mathrm{~Hz}, 50 \mathrm{~ms}$ tone with a $5 \mathrm{~ms}$ rise/fall time at $62 \mathrm{~dB}$ ), and $10 \%$ were novel stimuli (visual: fractal images; auditory: man-made and natural sounds) that were, on average, $250 \mathrm{~ms}$ in duration and presented at $62 \mathrm{~dB}$ (66). All visual stimuli were presented for $500 \mathrm{~ms}$ and the difference in radius between the target and standard circle was $\sim 104: 67$ in ratio. Stimuli were presented in the same pseudorandom order for all participants. Target and novel stimuli were not allowed to repeat in a sequence such that two deviant stimuli could not occur in a row.

Participants were instructed to respond to the target stimulus and withhold a response to both standard and novel stimuli. Participants indicated their response by pressing a button on a Cedrus ${ }^{\circledR}$ response box using the index finger of their dominant hand. Incorrect trials were excluded from EEG analysis. There was a fixed, 1,250 ms stimulus onset asynchrony 
TABLE 1 | Participant demographics.

\begin{tabular}{|c|c|c|c|c|c|c|c|}
\hline \multirow[b]{2}{*}{ Group } & \multirow[b]{2}{*}{$\mathbf{N}$} & \multirow[b]{2}{*}{ Age (SD) } & \multirow[b]{2}{*}{ Females (\%) } & \multicolumn{4}{|c|}{ Mean SOPS scores (SD) } \\
\hline & & & & Positive & Negative & Disorganized & General \\
\hline Conv-/ASD- (non-converter) & 219 & $19.56(4.63)$ & $101(46.11)$ & $11.60(4.23)$ & $11.71(6.14)$ & $4.87(3.23)$ & $8.96(4.29)$ \\
\hline Conv+/ASD- (converter) & 71 & $18.79(3.67)$ & $29(40.85)$ & $13.54(3.84)$ & $12.10(6.48)$ & $6.31(3.75)$ & $9.52(4.40)$ \\
\hline Conv-/ASD+ (non-converter) & 10 & $17.28(2.91)$ & $1(10)$ & $13.80(3.23)$ & $13.80(4.49)$ & $6.80(2.62)$ & $8.40(5.70)$ \\
\hline Conv+/ASD+ (converter) & 4 & $15.98(2.56)$ & $0(0)$ & $12.25(3.10)$ & $10.50(5.51)$ & $5.75(5.50)$ & $8.25(0.96)$ \\
\hline
\end{tabular}

(SOA) between auditory oddball trials such that each block lasted approximately $3 \mathrm{~min}$. Visual oddball trials were jittered between 1,500 and $2,500 \mathrm{~ms}$ (mean $\mathrm{SOA}=2 \mathrm{~s}$ ) to avoid simultaneous presentation with auditory stimuli from a background mismatch negativity task. Stimulus presentation was implemented with Presentation ${ }^{\circledR}$ software (Version 13.0, Neurobehavioral Systems, Inc., Berkeley, CA, www.neurobs.com).

\section{Electroencephalographic Data Acquisition and Pre-processing}

Participants sat in front of a computer monitor with a screen resolution of $1,024 \times 768$ and a refresh rate of $60 \mathrm{~Hz}$. As described in (32), EEG was recorded at $1024 \mathrm{~Hz}$ using either a 32-channel (4 NAPLS2 sites) or 64-channel (remaining 4 sites) BioSemi ActiveTwo recording system (BioSemi, Amsterdam, Netherlands). Additional electrodes were placed on the nose and mastoids with vertical electrooculogram (VEOG) recorded at electrodes placed above and below the right eye and horizontal (HEOG) electrodes at the outer canthus of each eye.

Continuous EEG data were re-referenced to averaged mastoids and high-pass filtered $(0.1 \mathrm{~Hz})$. Data were then processed using a modified version [see (32) for detail] of the Fully Automated Statistical Thresholding for EEG artifact Rejection (FASTER) Routine (67), with additional modification of ICA component selection as per previous literature [see (68)] to ensure proper removal of visual artifacts in the visual oddball task where blinks and saccades may be temporally correlated with ERP components. Continuous EEG data were segmented from $-1,000$ to 2,000 ms time-locked to the onset of the stimulus during FASTER pre-processing. Last, ERP data were baseline corrected ( -100 to $0 \mathrm{~ms}$ ) and low-passed filtered at $30 \mathrm{~Hz}$.

\section{Statistical Analysis}

The measure of interest in the oddball task was P300 amplitude, which was disambiguated by computing difference waveforms by subtracting the standard ERP form target (P3b) and novel (P3a) ERPs separately for the auditory and visual tasks. P300 amplitude was defined based on previous literature $(32,69)$ as the peak amplitude, elicited between 235 and $400 \mathrm{~ms}$ follow stimulus onset for auditory stimuli and 230-500 ms for visual stimuli. Peak amplitude was identified within each of these windows at $\mathrm{Cz}$ for $\mathrm{P} 3 \mathrm{a}$ (in response to task-irrelevant, non-target stimuli) and at $\mathrm{Pz}$ for $\mathrm{P} 3 \mathrm{~b}$ (in response to target stimuli), based on previous literature showing these sites are where $\mathrm{P} 3 \mathrm{a}$ and $\mathrm{P} 3 \mathrm{~b}$, respectively, have maximal amplitude. Average amplitude value within a $30 \mathrm{~ms}$ window centered around this peak was extracted. Thereafter, a
TABLE 2 | Oddball behavioral data ANOVA summary.

\begin{tabular}{|c|c|c|c|c|}
\hline & df & $\mathbf{F}$ & $p$ & $\eta_{\mathrm{p}}^{2}$ \\
\hline \multicolumn{5}{|l|}{ Accuracy } \\
\hline Modality & 1,300 & 0.26 & 0.61 & 0.001 \\
\hline Conversion status & 1,300 & 0.21 & 0.65 & 0.001 \\
\hline ASD status & 1,300 & 0.10 & 0.75 & $<0.001$ \\
\hline Modality $\times$ Conversion & 1,300 & 0.87 & 0.35 & 0.003 \\
\hline Modality $\times$ ASD & 1,300 & 0.047 & 0.83 & $<0.001$ \\
\hline Conversion $\times$ ASD & 1,300 & 0.14 & 0.71 & $<0.001$ \\
\hline Conversion $\times$ ASD $\times$ Modality & 1,300 & 0.028 & 0.87 & $<0.001$ \\
\hline \multicolumn{5}{|l|}{ Reaction time } \\
\hline Modality & 1,300 & $<0.001$ & 1.00 & $<0.001$ \\
\hline Conversion status & 1,300 & 0.009 & 0.93 & $<0.001$ \\
\hline ASD status & 1,300 & 1.62 & 0.93 & $<0.001$ \\
\hline Modality $\times$ Conversion & 1,300 & 0.88 & 0.35 & 0.003 \\
\hline Modality $\times$ ASD & 1,300 & 0.44 & 0.51 & 0.001 \\
\hline Conversion $\times$ ASD & 1,300 & 2.83 & 0.20 & 0.005 \\
\hline Conversion $\times$ ASD $\times$ Modality & 1,300 & 0.46 & 0.83 & $<0.001$ \\
\hline
\end{tabular}

statistical correction was applied to all ERP measures to adjust for normal aging effects and data collection site (32). In short, the age-corrected P300 amplitude z-score describes the amount, in standard units, that a participant's amplitude deviates from the value expected for a healthy individual of a given age assessed at a specific consortium site (70-72).

A binomial logistic regression model was applied to examine whether the relationship between baseline P300 amplitude and later psychosis conversion status (Conv+, Conv-) was moderated by whether or not individuals had a prior ASD diagnosis. The effects of interest were the main effect of P300 amplitude and the interaction term between ASD and P300 amplitude. Separate models were used for the four conditions (modality: auditory, visual; stimulus type: target, novel) to prevent collinearity of predictors. Main effects of amplitude would replicate prior findings showing that P300 predicts conversion to psychosis in CHR samples. A statistically significant ASD $\times$ P300 amplitude interaction would suggest the association between P300 amplitude and converting to psychosis changes based on ASD status. The ASD+ group was used as the reference condition, and bootstrapping procedures with 1,000 resamples were used to assess statistical significance and model stability. When significant interactions between P300 amplitude and ASD diagnosis were present, follow up analyses with simple 

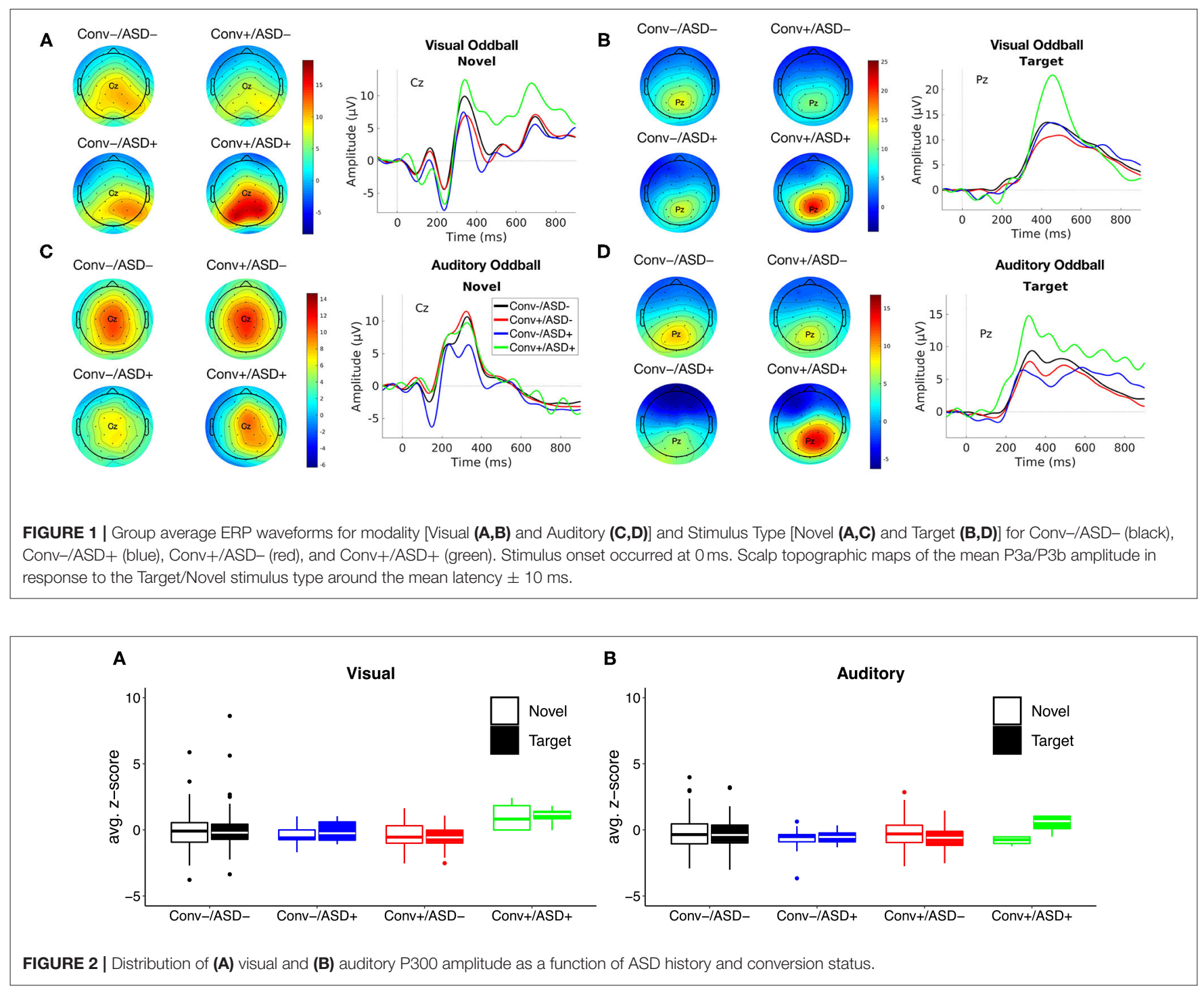

slopes were computed to better understand the moderating effect of ASD.

To ensure any neural differences detected weren't simply downstream effects of differing behavioral performance across participants, behavioral measures of accuracy and reaction time of target detection were analyzed separately using a 3-way [ASD diagnosis (ASD+, ASD-) $\times$ Conversion status (Conv+, Conv-) $\times$ Modality (auditory, visual)] repeated measures analysis of variance (ANOVA). Accuracy was calculated as percent correct and comprised total hits [i.e., responding to $(70,71)$ the target] and correct rejections (i.e., withholding a response to novel, task-irrelevant stimuli and frequent standards) given as the

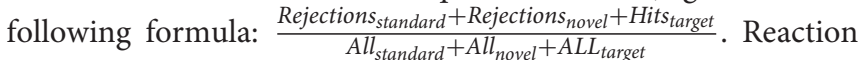
time reflected the time taken to press the button (i.e., respond) to Target stimuli in each modality.

Significance testing was conducted with an alpha level of $p=0.05$, with $p$-values generated from bootstrapping with 1,000 resamples. Since this study was designed to be hypothesis-generating given the small number of individuals with ASD history in the NAPLS2 cohort, we did not correct for multiple comparisons in order to reduce the chance of type two error.

\section{RESULTS}

\section{Oddball Behavioral Data}

Behavioral performance is summarized in Table 2. The analysis of response accuracy revealed no significant main effects or interactions between ASD and conversion status, and all participants were highly accurate. Results indicated that, across groups, participants were equally accurate on the visual [ $(0.99$ $\pm 0.008)$, (Mean \pm SE) $]$ as they were on the auditory $(0.98 \pm$ $0.006)$ oddball task $\left(F_{1,300}=0.26, p=0.61\right)$. There were no differences in accuracy between groups based on Conversion status $\left(\mathrm{F}_{1,300}=0.65, p=0.646\right.$; Conv-: $0.99 \pm 0.006$; Conv+: $0.98 \pm 0.009)$ or ASD diagnosis $\left(\mathrm{F}_{1,300}=0.10, p=0.75\right.$; ASD-: 
TABLE 3 | Binomial logistic regression summary with bootstrapping of oddball ERP data.

\begin{tabular}{lcccc}
\hline Model & B & SE & OR & $\boldsymbol{p}$ \\
\hline Visual - Novel & & & & \\
P3a Amplitude & 1.47 & 0.78 & 4.36 & 0.004 \\
Amplitude $\times$ ASD Diagnosis & -1.80 & 0.80 & - & 0.001 \\
Visual - Target & & & & \\
P3b Amplitude & 1.56 & 0.72 & 4.73 & 0.004 \\
Amplitude $\times$ ASD Diagnosis & -2.16 & 0.75 & - & 0.001 \\
Auditory - Novel & & & & 0.78 \\
P3a Amplitude & -0.12 & 0.47 & 0.89 & 0.80 \\
Amplitude $\times$ ASD Diagnosis & 0.12 & 0.48 & - & \\
Auditory - Target & & & & 0.045 \\
P3b Amplitude & 2.98 & 1.54 & 19.70 & 0.039 \\
Amplitude $\times$ ASD Diagnosis & -3.37 & 1.56 & - & \\
\hline
\end{tabular}

$0.98 \pm 0.002 ; \mathrm{ASD}+: 0.99 \pm 0.01)$. Analysis of reaction time also showed no significant main or interaction effects (see Table 2). Across conversion status (Conv-: $486.35 \pm 13.70 \mathrm{~ms}$; Conv+: $483.94 \pm 21.78 \mathrm{~ms}$ ), ASD diagnosis (ASD-: $501.51 \pm 5.78 \mathrm{~ms}$; ASD+: $468.78 \pm 25.07 \mathrm{~ms}$ ), and modality (Auditory: $485.15 \pm$ $15.25 \mathrm{~ms}$; Visual: $485.15 \pm 13.50 \mathrm{~ms}$ ), groups were comparable in their reaction time.

\section{ERP Data}

There were no significant differences in the number of included ERP trials between groups, overall or as a function of stimulus type or modality (Conversion: $\mathrm{F}_{1,300}=0.52, p=0.47$; ASD: $\mathrm{F}_{1,300}$ $=0.007, p=0.94$; Conversion $\times$ ASD: $\mathrm{F}_{1,300}=0.63, p=0.63 ; 2$ way interactions with Conversion: $p>0.05 ; 2$-way interactions with ASD: $p>0.05$; 3 - and 4-way interaction with Conversion and ASD status: $p>0.05$ ).

Our central question was whether the predictive relationship between P300 amplitude and conversion status was moderated by history of ASD diagnosis. Figure 1 shows waveforms by modality and condition, as a function of ASD and Conversion status. Figures 2A,B show z-score corrected P300 amplitudes for auditory and visual modalities, respectively. The estimated regression parameters are summarized in Table 3. A main effect of P300 amplitude predicting conversion status was significant in the visual modality (P3a: $p=0.004$; P3b: $p=0.004$ ) and in the auditory modality for P3b $(p=0.045)$, but not P3a $(p=0.78)$.The ASD $\times$ P300 Amplitude interaction significantly predicted conversion status in models of both Novel (P3a: $p=$ 0.001 ) and Target (P3b: $p=0.001$ ) stimuli in the visual modality, and for Target stimuli (P3b) in the auditory modality $(p=0.039)$, but not for auditory novel stimuli (P3a: $p=0.80)$.

Simple slopes analyses indicated that, within the ASD+ group, more enhanced P300 amplitudes (relative to the TD sample against which they were z-scored) were significantly associated with conversion to psychosis for both auditory and visual target stimuli, as well as for visual novel stimuli (Auditory P3b: OR $=16.72, \beta=2.82, \mathrm{SE}=1.49, p=0.011$; Visual P3b: $\mathrm{OR}=$ $7.80, \beta=2.05, \mathrm{SE}=1.21, p=0.025$; Visual P3a: $\mathrm{OR}=4.47$, $\beta=1.50, \mathrm{SE}=0.83, p=0.008$ ). There was no association between Auditory P3a amplitude and psychosis conversion in the ASD + group $(p=0.93)$. See Figure 3 for individual waveforms by condition from all four Conv+/ASD+ participants. These findings contrast with the ASD- CHR subset, wherein P300 enhancements were significantly associated with decreased risk of conversion to psychosis (Auditory P3b: $\mathrm{OR}=0.67, \beta=-0.39$, $\mathrm{SE}=0.16, p=0.006$; Visual P3a: $\mathrm{OR}=0.72, \beta=-0.32$, $\mathrm{SE}$ $=0.13, p=0.015$; Visual P3b: OR $=0.55, \beta=-0.60, \mathrm{SE}=$ $0.17, p=0.001$ ), consistent with previous literatures wherein attenuated P300 amplitudes typically associate with conversion to psychosis.

\section{DISCUSSION}

In this paper, we present exploratory analyses of the utility of EEG markers for predicting conversion to psychosis in a unique, albeit small, sample of individuals with ASD at clinical high risk for psychosis, followed longitudinally for two years. We find that P300 amplitude profiles to visual target and novel and auditory target stimuli in CHR patients differentially predict conversion to psychosis as a function of ASD status. In the general CHR population, previous literature shows that reduced $\mathrm{P} 300$, and particularly $\mathrm{P} 3 \mathrm{~b}$ response to behaviorallyrelevant auditory stimuli, is both characteristic of the group as a whole and predictive of later conversion to psychosis. Here, we find preliminary evidence that history of ASD diagnosis moderates this relationship. In particular, enhanced - rather than attenuated - P300 response to visual and target stimuli appears to be a unique profile associated with conversion to psychosis among CHR individuals with ASD history. Whereas, intact or enhanced $\mathrm{P} 300$ response is typically a positive prognostic marker in the CHR literature, we show that, for every one standard deviation increase in P300 amplitude above the mean in healthy controls, CHR individuals with ASD history have between 4 and 16 times greater chance of developing psychosis. Such pattern was not characteristic of either CHR individuals without ASD, or CHR individuals with ASD who did not convert to psychosis. Moreover, the observed odds ratios for P300 predicting conversion to psychosis in ASD are strikingly large compared to those often seen in studies examining predictors of psychosis in broader CHR groups. Neither accuracy nor reaction time during task performance differed between $\mathrm{CHR}$ patients with ASD who converted to psychosis and any of the other study groups, and the amount of data retained for analysis also did differ by group. These factors contribute to early confidence that observed differences in ERP response likely reflect true differences in brain response, rather than being artifactual as a function of differences in behavior response patterns or data quality.

Our oddball task findings in CHR patients without ASD align with previous work showing that P300 amplitude is reduced in psychosis $(18,19)$, in $\operatorname{CHR}(29,30,73)$, and in those with CHR who convert to psychosis $(32,33,60,74)$. In patients with CHR who have a prior ASD history but do not convert to psychosis, we also see $\mathrm{P} 300$ reductions that are consistent 


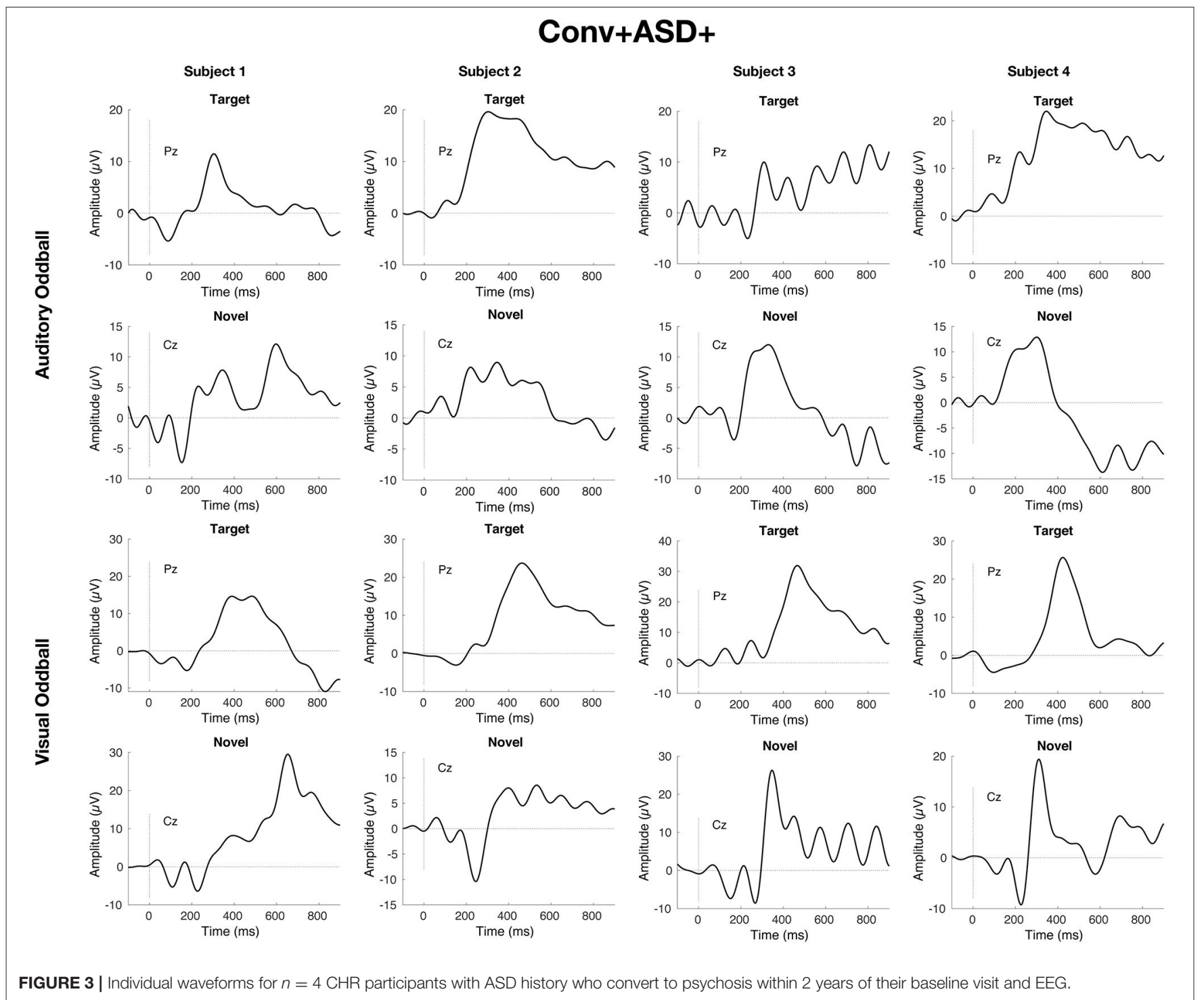

with both the broader CHR group and the general literature on P300 in ASD without psychosis $(61,75)$. Indeed, the pattern of enhanced P300 to visual and target stimuli appears to be unique to those with CHR and ASD whose illness trajectory results in full-blown psychosis within 2 years. It may reflect allocation of an aberrantly large degree of attention to sensory input, in the visual domain, regardless of behavioral relevance of stimuli, as well as when sensory input is behaviorally relevant, regardless of sensory domain. Of note, as accuracy of responding to target stimuli did not differ among participants as a function of ASD or conversion status, post-attention decision making steps may still function similarly, at least in the context of a simple detection task, despite differential attentional allocation at the neural level.

These results provide initial evidence suggesting that ASD status may be important to account for when evaluating neural markers that may predict later transition to psychosis in CHR individuals. This finding is interesting in light of the fact that clinical predictors of conversion to psychosis do not seem distinct in CHR individuals with ASD vs. those without (17), suggesting additive information from the neural data. Based on prior literature (32), more attenuated P300 amplitude in CHR individuals ought to raise greater concern about future conversion to psychosis. Thus, with such literature as background and without knowledge of prior ASD status, discovering enhanced P300 amplitude to oddball stimuli in a CHR individual might be cause for optimism about prognosis and recovery. If borne out in larger studies, our results suggest that knowing the ASD history of these individuals may therefore be of import: only if one know the individual's prior ASD diagnosis can one make the more nuanced interpretation, raising concern about conversion as a function of the P300 enhancement. Combined with clinical and demographic indicators of risk for conversion to psychosis, this information from EEG could in turn contribute to more accurate predictions about disease trajectory.

Study findings are of course limited by our small sample of CHR individuals with ASD, particularly for those who 
converted to psychosis. However, the large odds ratios we uncovered support the import of this hypothesis-generating work. In addition, our sample consists only of help-seeking individuals, who are plausibly not entirely representative of the broader population of those with ASD and psychoticlike symptoms. Finally, CHR itself is a broad category, and the range of concerning symptoms expressed at baseline was likely heterogenous both within our ASD subset and within the broader CHR group. Due to our small sample size, we did not look at individual clinical symptom associations, but baseline symptoms did not differ among those with or without ASD, or who did or did not convert to psychosis. Despite study limitations, the striking dissociation among groups that we discovered provides an exemplar of why this line of work is critically important, as our findings would be entirely masked were ASD status not considered. Samples of ASD individuals with CHR symptoms followed longitudinally are exceedingly rare to date, making our findings important, even if preliminary. Future studies in larger samples of $\mathrm{CHR}$ individuals with ASD and comparing to non-CHR ASD are needed in order to validate our preliminary findings and ensure they are not spurious. Should they replicate in larger samples, our results could mean new insight into prevention and intervention in patients presenting to CHR clinics with ASD history, and for those presenting to ASD clinics with early signs of psychosis.

\section{DATA AVAILABILITY STATEMENT}

The data analyzed in this study is subject to the following licenses/restrictions: data belongs to the NAPLS2 consortium and may be available upon request. Requests to access these datasets should be directed to daniel.mathalon@ucsf.edu.

\section{ETHICS STATEMENT}

Study procedures were reviewed by and approved across all eight sites of the NAPLS2 consortium. Written informed consent to participate in this study was provided by the participant, or by the participant's legal guardian for those under 18 years of age.

\section{REFERENCES}

1. Crespi B, Stead P, Elliot M. Evolution in health and medicine Sackler colloquium: comparative genomics of autism and schizophrenia. Proc Natl Acad Sci USA. (2010) 107 (Suppl. 1):1736-41. doi: 10.1073/pnas.0906080106

2. Fusar-Poli P, Politi P. Paul Eugen Bleuler and the birth of schizophrenia. (1908). Am J Psychiatry. (2008) 165:1407. doi: 10.1176/appi.ajp.2008.08050714

3. American Psychiatric Pub. Diagnostic and Statistical Manual of Mental Disorders (DSM-5®). American Psychiatric Pub (2013).

4. Marwick K, Hall J. Social cognition in schizophrenia: a review of face processing. Br Med Bull. (2008) 88:43-58. doi: 10.1093/bmb/ldn035

5. Mouridsen SE, Rich B, Isager T. Psychiatric disorders in adults diagnosed as children with atypical autism. A case control study. J Neural Trans. (2008) 115:135-8. doi: 10.1007/s00702-007-0798-1

\section{AUTHOR CONTRIBUTIONS}

JF-F, EV, PB, AB, GL, MN, KC, TM, DP, LS, SW, TC, and DM: concept and design. JF-F, SG, BR, HH, PB, RC, ED, JJ, GL, MN, JA, CB, KC, BC, LS, WS, MT, EW, SW, TC, and DM: acquisition, analysis, or interpretation of data. JF-F, SG, BR, HH, and DM: drafting of the manuscript. JF-F, SG, BR, EV, HH, PB, AB, RC, ED, JJ, GL, MN, JA, CB, KC, BC, TM, DP, WS, MT, EW, SW, TC, and DM: critical revision of the manuscript for important intellectual content. JF-F, SG, BR, and DM: statistical analysis. JA, $\mathrm{CB}, \mathrm{KC}, \mathrm{BC}, \mathrm{DP}, \mathrm{LS}, \mathrm{EW}, \mathrm{TC}$, and DM: obtained funding. $\mathrm{HH}$, BR, PB, RC, ED, GL, MN, JA, CB, KC, BC, LS, WS, EW, TC, and DM: administrative, technical, or material support. All authors contributed to the article and approved the submitted version.

\section{FUNDING}

This work was supported by the National Institute of Mental Health (Grant U01MH081984 to JA; Grants U01 MH081928; P50 MH080272; Commonwealth of Massachusetts SCDMH82101008006 to LS; Grants R01 MH60720, U01 MH082022, and K24 MH76191 to KC; Grant MH081902 and U01MH081902 to TC; Grant P50 MH066286 and support from the Staglin Family Music Festival for Mental Health to CB; Grant U01MH076989 to DM; Grant U01MH082004-01A1 to DP; Grant U01MH081988 to EW; Grant U01MH082022 to SW; and U01 MH081857-05 Grant to BC). ED receives or has received research support for work unrelated to this project from NIMH (1R01MH117315-01A1; 5R21MH117512-02), the Department of Veterans Affairs (1I01CX000974-01A1), Auspex Pharmaceuticals, Inc. and Teva Pharmaceuticals, Inc. ED was a full-time attending psychiatrist in the Mental Health Service Line at the Atlanta Veterans Affairs Health Care System, Decatur, GA. The content is solely the responsibility of the authors and does not necessarily represent the official views of the Department of Veterans Affairs. JF-F was supported by National Institute of Mental Health R21 MH115297, R01 MH119172, and by the Seaver Foundation. EV received support from the Netherland Organization for Scientific Research (NWO) VENI Grant No. 916-15-005 and the Seaver Foundation. EV was a Seaver Faculty Scholar and SG was a Seaver Fellow. disorders and criminal behaviour: a case control study. Int $J$ Offender Ther Comp Criminol. (2008) 52:196-205. doi: 10.1177/0306624X07302056

7. Selten JP, Lundberg M, Rai D, Magnusson C. Risks for nonaffective psychotic disorder and bipolar disorder in young people with autism spectrum disorder: a population-based study. JAMA Psychiatry. (2015) 72:483-9. doi: 10.1001/jamapsychiatry.2014.3059

8. Kincaid DL, Doris M, Shannon C, Mulholland C. What is the prevalence of autism spectrum disorder and ASD traits in psychosis? A systematic review. Psychiatry Res. (2017) 250:99-105. doi: 10.1016/j.psychres.2017.01.017

9. Hafner H, Loffler W, Maurer K, Hambrecht M, der Heiden W. Depression, negative symptoms, social stagnation and social decline in the early course of schizophrenia. Acta Psychiatr Scand. (1999) 100:105-18. doi: 10.1111/j.1600-0447.1999.tb10831.x 
10. Keefe RS, Eesley CE, Poe MP. Defining a cognitive function decrement in schizophrenia. Biol Psychiatry. (2005) 57:688-91. doi: 10.1016/j.biopsych.2005.01.003

11. Mayes SD, Calhoun SL. Learning, attention, writing, and processing speed in typical children and children with ADHD, autism, anxiety, depression, and oppositional-defiant disorder. Child Neuropsychol. (2007) 13:469-93. doi: 10.1080/09297040601112773

12. Nuechterlein $\mathrm{KH}$, Dawson ME. Information processing and attentional functioning in the developmental course of schizophrenic disorders. Schizophr Bull. (1984) 10:160-203. doi: 10.1093/schbul/10.2.160

13. Cannon TD, Cadenhead K, Cornblatt B, Woods SW, Addington J, Walker E, et al. Prediction of psychosis in youth at high clinical risk: a multisite longitudinal study in North America. Arch Gen Psychiatry. (2008) 65:28-37. doi: 10.1001/archgenpsychiatry.2007.3

14. Cannon TD, Chung Y, He G, Sun D, Jacobson A, van Erp TGM, et al. Progressive reduction in cortical thickness as psychosis develops: a multisite longitudinal neuroimaging study of youth at elevated clinical risk. Biol Psychiatry. (2015) 77:147-57. doi: 10.1016/j.biopsych.2014.05.023

15. Dazzan P, Soulsby B, Mechelli A, Wood SJ, Velakoulis D, Phillips LJ, et al. Volumetric abnormalities predating the onset of schizophrenia and affective psychoses: an MRI study in subjects at ultrahigh risk of psychosis. Schizophrenia Bull. (2012) 38:1083-91. doi: 10.1093/schbul/sbr035

16. Seidman LJ, Giuliano AJ, Meyer EC, Addington J, Cadenhead KS, Cannon TD, et al. Neuropsychology of the prodrome to psychosis in the NAPLS consortium: relationship to family history and conversion to psychosis. Arch Gen Psychiatry. (2010) 67:578-88. doi: 10.1001/archgenpsychiatry.2010.66

17. Foss-Feig JH, Velthorst E, Smith L, Reichenberg A, Addington J, Cadenhead $\mathrm{KS}$, et al. Clinical profiles and conversion rates among young individuals with autism spectrum disorder who present to clinical high risk for psychosis services. J Am Acad Child Adolesc Psychiatry. (2019) 58:582-8. doi: 10.1016/j.jaac.2018.09.446

18. Jeon YW, Polich J. Meta-analysis of P300 and schizophrenia: patients, paradigms, and practical implications. Psychophysiology. (2003) 40:684-701. doi: 10.1111/1469-8986.00070

19. Mathalon DH, Ford JM, Rosenbloom M, Pfefferbaum A. P300 reduction and prolongation with illness duration in schizophrenia. Biol Psychiatry. (2000) 47:413-27. doi: 10.1016/S0006-3223(99)00151-1

20. Winterer G, Egan MF, Raedler T, Sanchez C, Jones DW, Coppola R, et al. P300 and genetic risk for schizophrenia. Arch Gen Psychiatry. (2003) 60:1158-67. doi: 10.1001/archpsyc.60.11.1158

21. Donchin E, Coles MG. Is the P300 component a manifestation of context updating? Behav Brain Sci. (1988) 11:357-74. doi: 10.1017/S0140525X00058027

22. Duncan-Johnson CC, Donchin E. On quantifying surprise: the variation of event-related potentials with subjective probability. Psychophysiology. (1977) 14:456-67. doi: 10.1111/j.1469-8986.1977.tb01312.x

23. Isreal JB, Wickens CD, Chesney GL, Donchin E. The event-related brain potential as an index of display-monitoring workload. Human Fact. (1980) 22:211-24. doi: 10.1177/001872088002200210

24. Johnson RJr. A triarchic model of P300 amplitude. Psychophysiology. (1986) 23:367-84. doi: 10.1111/j.1469-8986.1986.tb00649.x

25. Knight RT. Evoked potential studies of attention capacity in human frontal lobe lesions. In: Levin H, Eisenberg H, Benton F editors. Frontal Lobe Function and Dysfunction. Oxford: Oxford University Press (1991). p. 139-53.

26. Polich J. Habituation of $\mathrm{P} 300$ from auditory stimuli. Psychobiology. (1989) 17:19-28.

27. Sutton S, Tueting $\mathrm{P}$, Zubin J, John ER. Information delivery and the sensory evoked potential. Science. (1967) 155:1436-9. doi: $10.1126 /$ science.155.3768.1436

28. Luck SJ, Mathalon DH, O'Donnell BF, Hamalainen MS, Spencer KM, Javitt DC, et al. A roadmap for the development and validation of event-related potential biomarkers in schizophrenia research. Biol Psychiatry. (2011) 70:2834. doi: 10.1016/j.biopsych.2010.09.021

29. del Re EC, Spencer KM, Oribe N, Mesholam-Gately RI, Goldstein J, Shenton ME, et al. Clinical high risk and first episode schizophrenia: auditory event-related potentials. Psychiatry Res. (2015) 231:126-33. doi: 10.1016/j.pscychresns.2014.11.012
30. van der Stelt O, Lieberman JA, Belger A. Auditory P300 in high-risk, recent-onset and chronic schizophrenia. Schizophr Res. (2005) 77:309-20. doi: 10.1016/j.schres.2005.04.024

31. Hamilton HK, Boos A, Mathalon DH. Electroencephalography and eventrelated potential biomarkers in individuals at clinical high risk for psychosis. Biol Psychiatry. (2020) 88:294-303. doi: 10.1016/j.biopsych.2020.04.002

32. Hamilton HK, Roach BJ, Bachman PM, Belger A, Carrion RE, Duncan E, et al. Association between P300 responses to auditory oddball stimuli and clinical outcomes in the psychosis risk syndrome. JAMA Psychiatry. (2019) 76:1187-97. doi: 10.1001/jamapsychiatry.2019.2135

33. Hamilton HK, Woods SW, Roach BJ, Llerena K, McGlashan TH, Srihari VH, et al. Auditory and visual oddball stimulus processing deficits in schizophrenia and the psychosis risk syndrome: forecasting psychosis risk with P300. Schizophr Bull. (2019) 45:1068-80. doi: 10.1093/schbul/sby167

34. Comerchero MD, Polich J. P3a, perceptual distinctiveness, and stimulus modality. Brain Res Cogn Brain Res. (1998) 7:41-8. doi: 10.1016/S0926-6410(98)00009-3

35. Courchesne E, Hillyard SA, Galambos R. Stimulus novelty, task relevance and the visual evoked potential in man. Electroencephalogr Clin Neurophysiol. (1975) 39:131-43. doi: 10.1016/0013-4694(75)90003-6

36. Goldstein A, Spencer KM, Donchin E. The influence of stimulus deviance and novelty on the P300 and novelty P3. Psychophysiology. (2002) 39:781-90. doi: 10.1111/1469-8986.3960781

37. Polich J. Updating P300: an integrative theory of P3a and P3b. Clin Neurophysiol. (2007) 118:2128-48. doi: 10.1016/j.clinph.2007.04.019

38. Squires NK, Squires KC, Hillyard SA. Two varieties of long-latency positive waves evoked by unpredictable auditory stimuli in man. Electroencephalogr Clin Neurophysiol. (1975) 38:387-401. doi: 10.1016/0013-4694(75)90263-1

39. Bramon E, Rabe-Hesketh S, Sham P, Murray RM, Frangou S. Meta-analysis of the P300 and P50 waveforms in schizophrenia. Schizophr Res. (2004) 70:315-29. doi: 10.1016/j.schres.2004.01.004

40. Demiralp T, Ucok A, Devrim M, Isoglu-Alkac U, Tecer A, Polich J. N2 and P3 components of event-related potential in first-episode schizophrenic patients: scalp topography, medication, and latency effects. Psychiatry Res. (2002) 111:167-79. doi: 10.1016/S0165-1781(02)00133-6

41. Ford JM, White PM, Csernansky JG, Faustman WO, Roth WT, Pfefferbaum A. ERPs in schizophrenia: effects of antipsychotic medication. Biol Psychiatry. (1994) 36:153-70. doi: 10.1016/0006-3223(94)91221-1

42. Pfefferbaum A, Ford JM, White PM, Roth WT. P3 in schizophrenia is affected by stimulus modality, response requirements, medication status, and negative symptoms. Arch Gen Psychiatry. (1989) 46:1035-44. doi: 10.1001/archpsyc.1989.01810110077011

43. Salisbury DF, Shenton ME, Sherwood AR, Fischer IA, Yurgelun-Todd DA, Tohen M, et al. First-episode schizophrenic psychosis differs from first-episode affective psychosis and controls in P300 amplitude over left temporal lobe. Arch Gen Psychiatry. (1998) 55:173-80. doi: 10.1001/archpsyc. 55.2 .173

44. Devrim-Ucok M, Keskin-Ergen HY, Ucok A. Novelty P3 and P3b in first-episode schizophrenia and chronic schizophrenia. Prog Neuropsychopharmacol Biol Psychiatry. (2006) 30:1426-34. doi: 10.1016/j.pnpbp.2006.05.019

45. Grillon C, Courchesne E, Ameli R, Geyer MA, Braff DL. Increased distractibility in schizophrenic patients. Electrophysiologic and behavioral evidence. Arch Gen Psychiatry. (1990) 47:171-9. doi: 10.1001/archpsyc.1990.01810140071010

46. Jahshan C, Cadenhead KS, Rissling AJ, Kirihara K, Braff DL, Light GA. Automatic sensory information processing abnormalities across the illness course of schizophrenia. Psychol Med. (2012) 42:85-97. doi: 10.1017/S0033291711001061

47. Light GA, Swerdlow NR, Thomas ML, Calkins ME, Green MF, Greenwood TA, et al. Validation of mismatch negativity and P3a for use in multi-site studies of schizophrenia: characterization of demographic, clinical, cognitive, and functional correlates in COGS-2. Schizophr Res. (2015) 163:63-72. doi: 10.1016/j.schres.2014.09.042

48. Mathalon DH, Hoffman RE, Watson TD, Miller RM, Roach BJ, Ford JM. Neurophysiological distinction between schizophrenia and schizoaffective disorder. Front Hum Neurosci. (2010) 3:70. doi: 10.3389/neuro.09.070.2009 
49. Merrin EL, Floyd TC. P300 responses to novel auditory stimuli in hospitalized schizophrenic patients. Biol Psychiatry. (1994) 36:527-42. doi: 10.1016/0006-3223(94)90617-3

50. Mondragón-Maya A, Solís-Vivanco R, León-Ortiz P, Rodríguez-Agudelo Y, Yáñez-Téllez G, Bernal-Hernández J, et al. Reduced P3a amplitudes in antipsychotic naive first-episode psychosis patients and individuals at clinical high-risk for psychosis. J Psychiatr Res. (2013) 47:755-61. doi: 10.1016/j.jpsychires.2012.12.017

51. Frodl T, Meisenzahl EM, Muller D, Greiner J, Juckel G, Leinsinger G, et al. Corpus callosum and P300 in schizophrenia. Schizophr Res. (2001) 49:107-19. doi: 10.1016/S0920-9964(00)00123-7

52. Schall U, Catts SV, Karayanidis F, Ward PB. Auditory event-related potential indices of fronto-temporal information processing in schizophrenia syndromes: valid outcome prediction of clozapine therapy in a three-year follow-up. Int J Neuropsychopharmacol. (1999) 2:83-93. doi: $10.1017 /$ S1461145799001418

53. Atkinson RJ, Michie PT, Schall U. Duration mismatch negativity and P3a in first-episode psychosis and individuals at ultra-high risk of psychosis. Biol Psychiatry. (2012) 71:98-104. doi: 10.1016/j.biopsych.2011.08.023

54. Bramon E, Shaikh M, Broome M, Lappin J, Berge D, Day F, et al. Abnormal P300 in people with high risk of developing psychosis. Neuroimage. (2008) 41:553-60. doi: 10.1016/j.neuroimage.2007.12.038

55. Frommann I, Brinkmeyer J, Ruhrmann S, Hack E, Brockhaus-Dumke A, Bechdolf A, et al. Auditory P300 in individuals clinically at risk for psychosis. Int J Psychophysiol. (2008) 70:192-205. doi: 10.1016/j.ijpsycho.2008. 07.003

56. Fusar-Poli P, Crossley N, Woolley J, Carletti F, Perez-Iglesias R, Broome M, et al. Gray matter alterations related to P300 abnormalities in subjects at high risk for psychosis: longitudinal MRI-EEG study. Neuroimage. (2011) 55:320-8. doi: 10.1016/j.neuroimage.2010.11.075

57. Fusar-Poli P, Crossley N, Woolley J, Carletti F, Perez-Iglesias R, Broome M, et al. White matter alterations related to P300 abnormalities in individuals at high risk for psychosis: an MRI-EEG study. J Psychiatry Neurosci. (2011) 36:239. doi: 10.1503/jpn.100083

58. Ozgurdal S, Gudlowski Y, Witthaus H, Kawohl W, Uhl I, Hauser M, et al. Reduction of auditory event-related P300 amplitude in subjects with at-risk mental state for schizophrenia. Schizophr Res. (2008) 105:272-8. doi: 10.1016/j.schres.2008.05.017

59. van der Stelt O, Frye J, Lieberman JA, Belger A. Impaired P3 generation reflects high-level and progressive neurocognitive dysfunction in schizophrenia. Arch Gen Psychiatry. (2004) 61:237-48. doi: 10.1001/archpsyc. 61.3.237

60. Van Tricht MJ, Nieman DH, Koelman JH, Van Der Meer JN, Bour LJ, De Haan L, et al. Reduced parietal P300 amplitude is associated with an increased risk for a first psychotic episode. Biol Psychiatry. (2010) 68:642-8. doi: 10.1016/j.biopsych.2010.04.022

61. Cui T, Wang PP, Liu S, Zhang X. P300 amplitude and latency in autism spectrum disorder: a meta-analysis. Eur Child Adolesc Psychiatry. (2017) 26:177-90. doi: 10.1007/s00787-016-0880-z

62. Addington J, Cadenhead KS, Cornblatt BA, Mathalon DH, McGlashan TH, Perkins DO, et al. North American Prodrome Longitudinal Study (NAPLS 2): overview and recruitment. Schizophr Res. (2012) 142:77-82. doi: 10.1016/j.schres.2012.09.012

63. Addington J, Liu L, Buchy L, Cadenhead KS, Cannon TD, Cornblatt BA, et al. North American Prodrome Longitudinal Study (NAPLS 2): the prodromal symptoms. J Nerv Ment Dis. (2015) 203:328-35. doi: 10.1097/NMD.0000000000000290
64. McGlashan T, Walsh B, Woods S. The Psychosis-Risk Syndrome: Handbook for Diagnosis and Follow-Up. New York, NY: Oxford University Press (2010).

65. Miller TJ, McGlashan TH, Rosen JL, Somjee L, Markovich PJ, Stein K, et al. Prospective diagnosis of the initial prodrome for schizophrenia based on the Structured Interview for Prodromal Syndromes: preliminary evidence of interrater reliability and predictive validity. Am J Psychiatry. (2002) 159:863-5. doi: 10.1176/appi.ajp.159.5.863

66. Friedman D, Simpson G, Hamberger M. Age-related changes in scalp topography to novel and target stimuli. Psychophysiology. (1993) 30:383-96.

67. Nolan H, Whelan R, Reilly RB. FASTER: Fully automated statistical thresholding for EEG artifact rejection. J Neurosci Methods. (2010) 192:152-62. doi: 10.1016/j.jneumeth.2010.07.015

68. Mognon A, Jovicich J, Bruzzone L, Buiatti M. ADJUST: an automatic EEG artifact detector based on the joint use of spatial and temporal features. Psychophysiology. (2011) 48:229-40. doi: 10.1111/j.1469-8986.2010.01061.x

69. Hamilton HK, Perez VB, Ford JM, Roach BJ, Jaeger J, Mathalon DH. Mismatch negativity but not P300 is associated with functional disability in schizophrenia. Schizophr Bull. (2018) 44:492-504. doi: 10.1093/schbul/sbx104

70. Perez VB, Ford JM, Roach BJ, Woods SW, McGlashan TH, Srihari $\mathrm{VH}$, et al. Error monitoring dysfunction across the illness course of schizophrenia. J Abnorm Psychol. (2012) 121:372-87. doi: 10.1037/a00 25487

71. Perez VB, Tarasenko M, Miyakoshi M, Pianka ST, Makeig SD, Braff DL, et al. Mismatch negativity is a sensitive and predictive biomarker of perceptual learning during auditory cognitive training in schizophrenia. Neuropsychopharmacology. (2017) 42:2206-13. doi: 10.1038/npp. 2017.25

72. Pfefferbaum A, Lim KO, Zipursky RB, Mathalon DH, Rosenbloom MJ, Lane B, et al. Brain gray and white matter volume loss accelerates with aging in chronic alcoholics: a quantitative MRI study. Alcohol Clin Exp Res. (1992) 16:1078-89. doi: 10.1111/j.1530-0277.1992.tb00702.x

73. Lee SY, Namkoong $\mathrm{K}$, Cho HH, Song D-H, An SK. Reduced visual P300 amplitudes in individuals at ultra-high risk for psychosis and first-episode schizophrenia. Neurosci Lett. (2010) 486:156-60. doi: 10.1016/j.neulet.2010.09.035

74. Tang Y, Wang J, Zhang T, Xu L, Qian Z, Cui H, et al. P300 as an index of transition to psychosis and of remission: data from a clinical high risk for psychosis study and review of literature. Schizophr Res. (2019) 226:74-83. doi: 10.1016/j.schres.2019.02.014

75. Courchesne E, Kilman BA, Galambos R, Lincoln AJ. Autism: processing of novel auditory information assessed by event-related brain potentials. Electroencephalogr Clin Neurophysiol Evoked Potent Sect. (1984) 59:238-48. doi: 10.1016/0168-5597(84)90063-7

Conflict of Interest: The authors declare that the research was conducted in the absence of any commercial or financial relationships that could be construed as a potential conflict of interest.

Copyright (C) 2021 Foss-Feig, Guillory, Roach, Velthorst, Hamilton, Bachman, Belger, Carrion, Duncan, Johannesen, Light, Niznikiewicz, Addington, Cadenhead, Cannon, Cornblatt, McGlashan, Perkins, Seidman, Stone, Tsuang, Walker, Woods, Bearden and Mathalon. This is an open-access article distributed under the terms of the Creative Commons Attribution License (CC BY). The use, distribution or reproduction in other forums is permitted, provided the original author(s) and the copyright owner(s) are credited and that the original publication in this journal is cited, in accordance with accepted academic practice. No use, distribution or reproduction is permitted which does not comply with these terms. 